\title{
Kathryn E. Merrick: Computational models of motivation for game-playing agents
}

Springer, 2016, 213 pp, ISBN: 978-3-319-33457-8

\section{Spyridon Samothrakis ${ }^{1}$}

Published online: 21 June 2018

(c) Springer Science+Business Media, LLC, part of Springer Nature 2018

Merrick's book helps game designers, programmers and researchers to create NonPlayer Characters (NPCs, i.e. agents) that have human like motives. She suggests that adding motives will create a richer and more life-like user experience for game players. Her book is very well written overall, and it would be of great interest to game developers, game designers and programmers.

It will introduce most of them to new concepts that range from game theory and multi-agent systems to the philosophy and psychology of power. It delivers what it sets out to do. After reading it you should be able to create more interesting characters for your game. Readers interested in evolutionary processes will recognise notions of co-evolution and evolutionary game theory; note that in line with the rest of the book, evolution is not introduced as an optimisation process in the strict sense, but rather as a tool for getting and/or understanding interesting (population) behaviour.

The book is split in four parts. Part I, which includes chapters 1, 2 and 3, discusses human motivation: how it can be modelled and how one can embed it in (artificial) agents. Part II, i.e. chapters 4 and 5, discuss how one can take inspiration from it and create individual profiles for motivated agents. Part II effectively applies methods from the previous chapters in a more applied context. Part III includes chapters 6, 7 and 8 and aims at providing useful scenarios for motivated agents as opponents, collaborators or story support characters. Part IV includes chapter 9 which discusses scenarios where motivated agents change their behaviour over time. Chapter 10 wraps up the book by providing further insights and proposing future directions, which include further things to read and further research

Spyridon Samothrakis

ssamot@essex.ac.uk

1 Institute of Analytics and Data Science, University of Essex, Colchester, UK 
to explore. I particularity enjoyed the introductory chapter, where Bartle's player types ${ }^{1}$ are linked to different motivational profiles. The follow-up chapter is also excellent and provides a coherent account of approach-avoidance, a theory of human psychology that aims to explain aspects of goal-seeking behaviour when the rewards of a goal are not entirely clear. Throughout the book the reader will find a wealth of modelling approaches and scenarios that can be used directly and applied to real computer games.

Although the ideas presented in the book are linked together, and there is considerable progression from chapter to chapter, I did not get a unique moment of clarity, were everything becomes obvious. One cannot discern a single abstract concept which everything emanates from. There is no clear way of compressing the chapters presented into a single idea; there is no grand unifying theme that the reader can fall back onto.

Another controversial aspect of the book is that, whenever it delves into actual player modelling using game theory, the computer agents (NPCs) do not take into account the reward structure of other agents/players, even when this seems somewhat counter-intuitive. For example, one can argue that pets should always have their owners best interests in mind. Therefore the game theory reward function should model how well they help their master/owner. However the book ignores this and instead tries to maximise the pet's own reward.

Concluding, Computational Models of Motivation for Game-Playing Agents will make a nice addition to the bookshelf of game designers, undergraduate students on computer game development courses and professional game researchers.

Acknowledgements I would like to thank Dr. William Langdon for the extensive and very helpful editing of the original review.

Publisher's Note Springer Nature remains neutral with regard to jurisdictional claims in published maps and institutional affiliations.

\footnotetext{
1 In 1996 Bartle created a taxonomy of player types that categorises human game players based on their reasons for playing games. For example, a socialising type might be motivated by power fantasies.
} 